\title{
Driving dynamics in the Formula Student
}

Marc Möckl, Christian Lichtenberg, Rennteam Uni Stuttgart e.V.

This manuscript is not available according to publishing restriction.

Thank you for your understanding. 